\title{
Olga Cantó Sánchez*
}

\section{DESIGUALDAD, REDISTRIBUCIÓN Y POLÍTICAS PÚBLICAS: ¿HAY UNA BRECHA GENERACIONAL?}

\begin{abstract}
Durante las últimas décadas se han registrado importantes cambios demográficos y socioeconómicos en los países industrializados que han aumentado notablemente el riesgo de vulnerabilidad y de pobreza de las generaciones más jóvenes. El objetivo de este trabajo es analizar qué papel desempeñan las políticas públicas en España en la lucha contra las desigualdades por cohortes de edad en un contexto comparado. Este análisis nos permitirá identificar cómo debemos avanzar en el diseño de políticas públicas que propicien un cambio de tendencia y mejoren la cohesión social desde un punto de vista intergeneracional.
\end{abstract}

\section{Inequality, redistribution and public policies: is there a generation gap?}

Recent decades have seen significant demographic and socioeconomic changes in industrialised countries which have significantly increased the risk of vulnerability and poverty among younger generations. The objective of this paper is to analyse the role played by public policies in Spain in the fight against inequalities by age cohorts in a comparative context. This analysis will allow us to identify how we should advance in the design of public policies that promote a change in this trend and improve social cohesion from an intergenerational point of view.

Palabras clave: desigualdad, pobreza, redistribución, jóvenes.

Keywords: inequality, poverty, redistribution, young people.

JEL: D63, I31.

\section{Introducción}

Los importantes cambios demográficos y socioeconómicos registrados durante las últimas décadas en los países industrializados han supuesto un notable aumento de la vulnerabilidad social de las generaciones más jóvenes con una clara tendencia al alza de la pobreza

\footnotetext{
* Universidad de Alcalá y EQUALITAS.

La autora desea agradecer la financiación recibida del Ministerio de Economía y Empresa (Proyecto ECO2016-76506-C4-2-R) para sus actividades de investigación, y la colaboración de Andrea Sobas Perela como ayudante de investigación para las simulaciones con Euromod I 1.0+. DOI: https://doi.org/10.32796/ice.2019.908.6826
}

infantil y juvenil en muchos países ricos. Esto es muy preocupante porque una amplia evidencia científica concluye que las consecuencias negativas de experimentar pobreza durante la infancia y la juventud tienden a persistir a lo largo de la vida del individuo (Ermisch et al., 2001), mermando las posibilidades de movilidad económica y social de la sociedad del futuro, lo que, a medio y largo plazo, puede propiciar un profundo deterioro en la igualdad de oportunidades vitales de la población (Corak et al., 2011). En general, la literatura más reciente sobre el tema subraya que la alta vulnerabilidad de los más jóvenes en los países desarrollados está fuertemente relacionada con la precariedad laboral, los bajos salarios y la 
inestabilidad en el empleo, lo que parece indicar que, a falta de políticas sociales correctoras, el proceso de deterioro de las condiciones laborales de las últimas décadas estaría detrás del progresivo aumento de las dificultades económicas de las nuevas generaciones.

No cabe duda de que la vulnerabilidad económica y la falta de recursos es el resultado de la compleja interacción entre factores económicos, demográficos y sociales, junto con varios elementos relacionados con la intervención pública, entre los que desempeñan un papel esencial tanto las políticas de empleo como el diseño y la intensidad protectora de las políticas impositivas y de transferencias públicas. Así, en un contexto de progresivo deterioro de las condiciones laborales y salariales, las políticas distributivas se vuelven clave en la determinación del bienestar económico.

En un amplio ejercicio a nivel europeo, De Agostini et al. (2016) y Bargain et al. (2017) constatan que los 27 países europeos son distintos en cuanto a la dimensión de los efectos redistributivos de sus sistemas de prestaciones e impuestos y que su evolución temporal a lo largo de la última década ha sido también diversa, dependiendo de la evolución de las rentas de mercado y de las reformas llevadas a cabo durante la recesión. En general, la evidencia empírica reciente apunta a que las políticas de austeridad en la Unión Europea han estado asociadas a aumentos en la desigualdad de renta disponible principalmente en la parte alta de la distribución (De Agostini et al., 2016; Paulus y Tasseva, 2018), pero en el caso de los países periféricos los aumentos en los ingresos públicos se consiguieron a través del aumento de impuestos personales y de consumo, más que aumentando impuestos sobre los beneficios o ganancias de capital. Como consecuencia, la recesión económica junto con la consolidación fiscal en España han impulsado el crecimiento de la desigualdad de la renta disponible y la reducción de la capacidad adquisitiva de muchos hogares modestos, colocados más bien en la cola baja de la distribución. Una parte importante de estos hogares están conformados por personas jóvenes con hijos dependientes cuyo riesgo de pobreza ha crecido mucho en la última década.
El objetivo de este artículo es analizar si en nuestro país hay una brecha generacional en el papel que desempeñan las políticas públicas en la lucha contra la vulnerabilidad y la pobreza. Para ello, analizaremos la capacidad de nuestro modelo de prestaciones e impuestos para redistribuir los ingresos y reducir el riesgo de pobreza de las personas que viven en distintos tipos de hogares en un contexto comparado. Parece natural compararnos con otros tres grandes países europeos con estados del bienestar de inspiración bismarckiana, es decir, basados como el nuestro en una idea de seguro para aquellos que han cotizado: Italia, Francia y Alemania; y también con Reino Unido, que tiene un sistema más asistencialista o «à la Beveridge», basado en cubrir situaciones de necesidad económica y teniendo mucho menos en cuenta las cotizaciones previas. Este análisis nos permitirá saber qué políticas están siendo las más efectivas para proteger a los hogares de distintas generaciones de la vulnerabilidad y la pobreza en distintos contextos, y nos dará algunas claves sobre cómo avanzar en el diseño de políticas públicas eficaces que permitan, a medio y largo plazo, cambiar la tendencia al alza de estos riesgos en las cohortes de edad más jóvenes.

La organización del texto es la siguiente: en el apartado 2 presentamos la evolución de la desigualdad y la pobreza en España y en los otros cuatro países europeos: Alemania, Francia, Italia y Reino Unido; medimos hasta qué punto esa desigualdad está ligada al papel redistributivo del sistema de prestaciones e impuestos, e identificamos cuáles son las políticas públicas que más comprimen la renta disponible en cada caso. En el apartado 3 valoramos si existe una brecha generacional en la capacidad que tienen los distintos estados del bienestar de este grupo de países para reducir la desigualdad y la vulnerabilidad económica. Para ello, calculamos en qué medida cada sistema redistribuye ingresos en tres tipos de hogares: los que están formados solo por menores de 40 años, los que incluyen a alguna persona menor de esa edad y los que incluyen solo a mayores de esa edad. El último apartado recoge las principales conclusiones del estudio. 


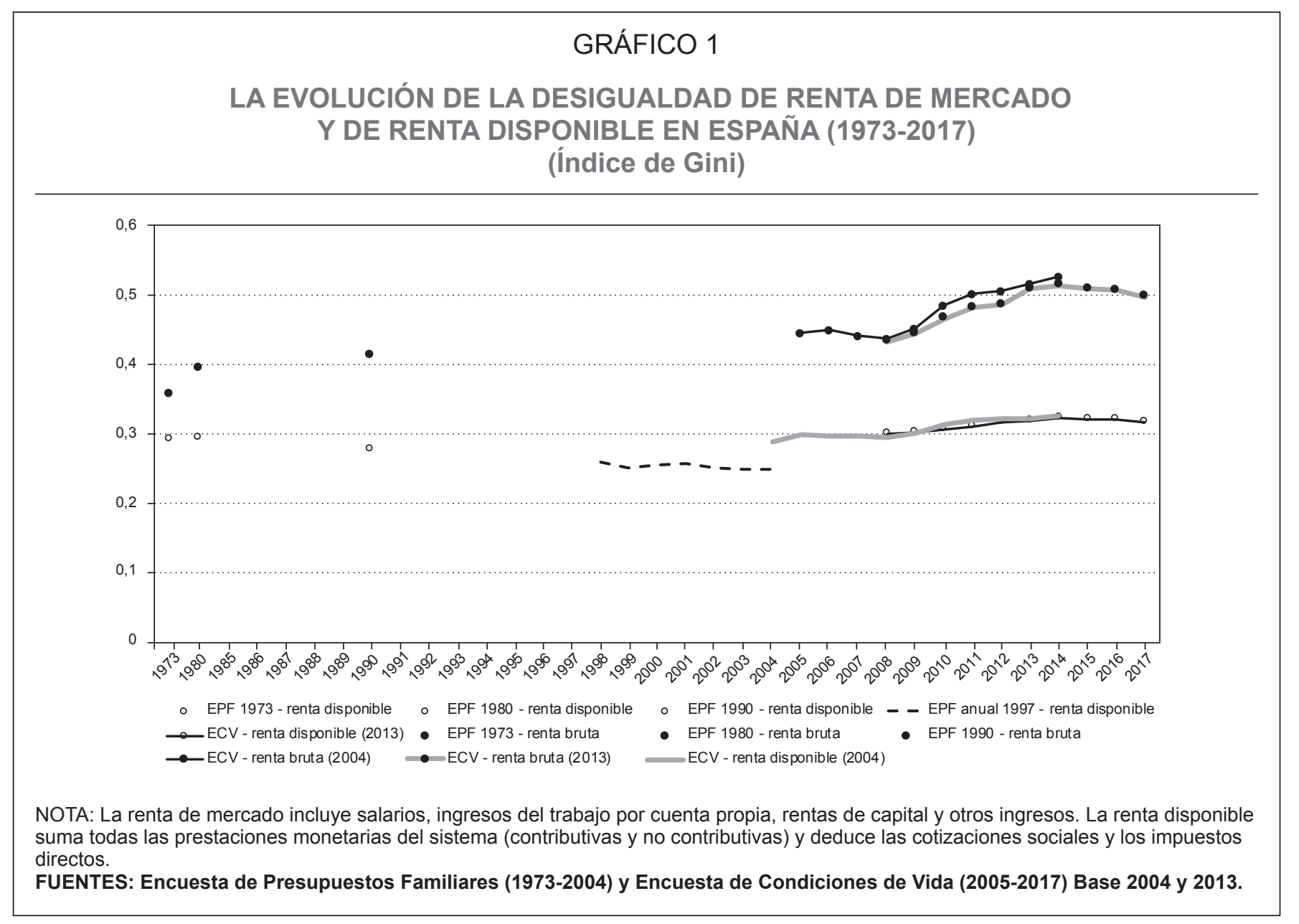

\section{La desigualdad y la pobreza en España en un contexto comparado}

Como han revelado multitud de informes recientes, España es uno de los países de la OCDE en los que la desigualdad y la pobreza han crecido más durante la recesión (OECD, 2015) y se coloca actualmente entre los cuatro países con mayor índice de Gini de la Unión Europea, solo por detrás de Bulgaria, Lituania y Letonia. Como subrayan Ayala y Cantó (2018) en un detallado análisis de la evolución de la desigualdad en España y sus determinantes, a principios de los años setenta nuestro país era uno de los Estados europeos que mayor brecha presentaba entre los ingresos del quintil más rico y los del quintil más pobre y eso sigue sucediendo hoy. Esta aparente estabilidad, sin embargo, esconde cambios muy importantes en la desigualdad de renta de mercado, esencialmente los salarios, y en el papel del sistema de impuestos y prestaciones que hace cuatro décadas redistribuía muy poco.

Dibujar un esquema de las tendencias de la desigualdad en España en un período largo de tiempo es una tarea difícil porque, a diferencia de otros países, no disponemos de información homogénea y sistemática sobre los ingresos de las familias españolas a lo largo del tiempo. A pesar de ello, y realizando un esfuerzo de armonización de las diferentes fuentes y teniendo presentes sus cambios metodológicos, el Gráfico 1 ofrece una panorámica interesante. De acuerdo con estos resultados, España no habría sufrido niveles de desigualdad 


\section{GRÁFICO 2}

\section{DESIGUALDAD DE RENTA DE MERCADO Y RENTA DISPONIBLE EN CINCO PAÍSES EUROPEOS (Índice de Gini)}
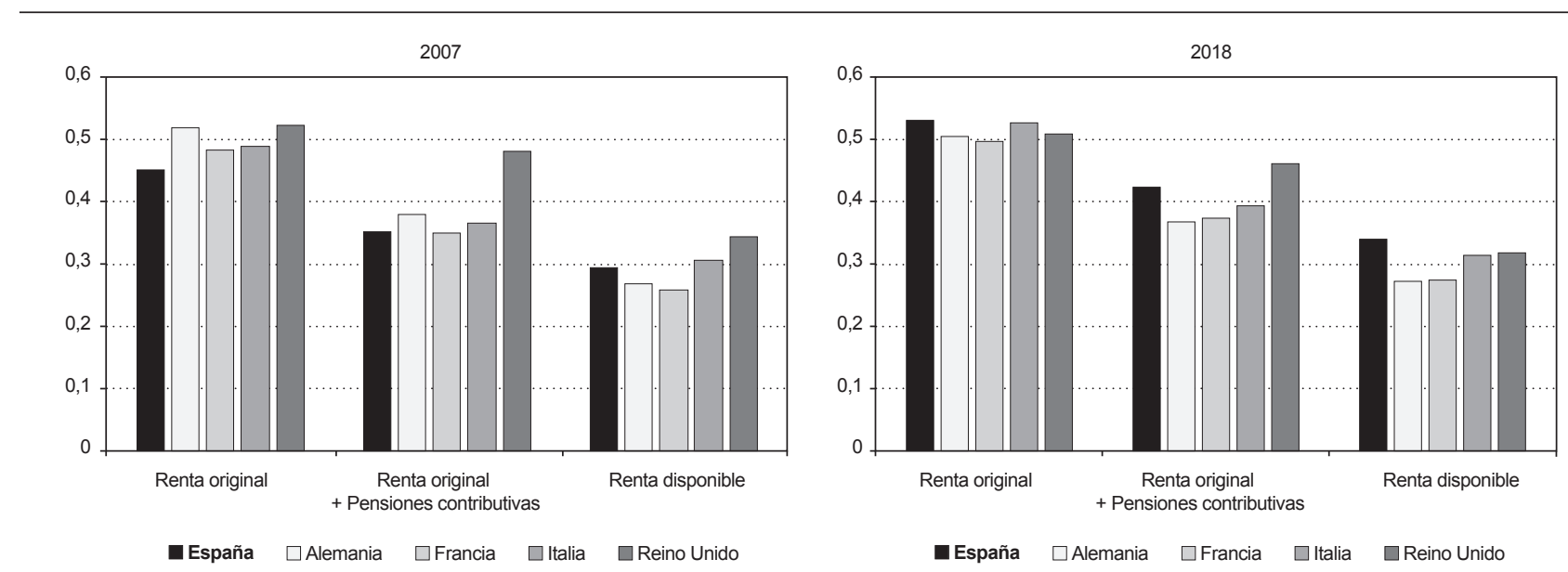

NOTA: La renta original incluye salarios, ingresos del trabajo por cuenta propia, rentas de capital y otros ingresos. La renta disponible incluye renta original y todas las prestaciones monetarias del sistema (contributivas y no contributivas) y deduce las cotizaciones sociales y los impuestos directos.

FUENTE: Euromod I 1.0+.

económica, ni extremadamente altos, ni muy alejados de los europeos, en los años setenta. Desde entonces, eso sí, han tenido lugar profundos cambios sociales y económicos que, además de colocarnos entre los países de renta alta en el contexto comparado, nos han permitido desarrollar un estado del bienestar que, aunque débil y con lagunas importantes, ha mejorado mucho su cobertura. De hecho, hoy tenemos un sistema que reduce las desigualdades de rentas de mercado más del doble de lo que lo hacía en 1973.

La mayor reducción de la desigualdad tuvo lugar durante los años ochenta, cuando se consiguió amortiguar el efecto del aumento del desempleo a través del desarrollo de las prestaciones y servicios de un incipiente estado del bienestar, que permitió que, al llegar la recuperación, este incremento del gasto social comprimiese la distribución de rentas, principalmente a través de la mejora del sistema de pensiones y de las prestaciones por desempleo.
La capacidad redistributiva de nuestro sistema creció hasta aproximadamente mediados de los noventa, pero desde entonces ha experimentado un cierto estancamiento. De hecho, la desigualdad de renta disponible fue tremendamente estable durante toda la primera década de este siglo, período de fuerte crecimiento económico y drástica reducción del desempleo, lo que solo se puede explicar si gran parte de los puestos de trabajo creados tenían remuneraciones bajas $\mathrm{y}$, al mismo tiempo, se producía un estancamiento de la capacidad redistributiva de las políticas públicas.

Si comparamos los niveles de desigualdad de renta disponible (Gráfico 2) de cinco grandes países europeos antes y después de la Gran Recesión, podemos comprobar que en 2007 nuestro país se colocaba en una posición intermedia, por debajo de Reino Unido e Italia y por encima de Francia y Alemania. Este resultado estaba claramente ligado a la mayor compresión de las rentas de mercado (salarios, ingresos del 


\section{GRÁFICO 3}

RIESGO DE POBREZA EN CINCO PAÍSES EUROPEOS

(En \% de población por debajo del umbral del $60 \%$ de la mediana)
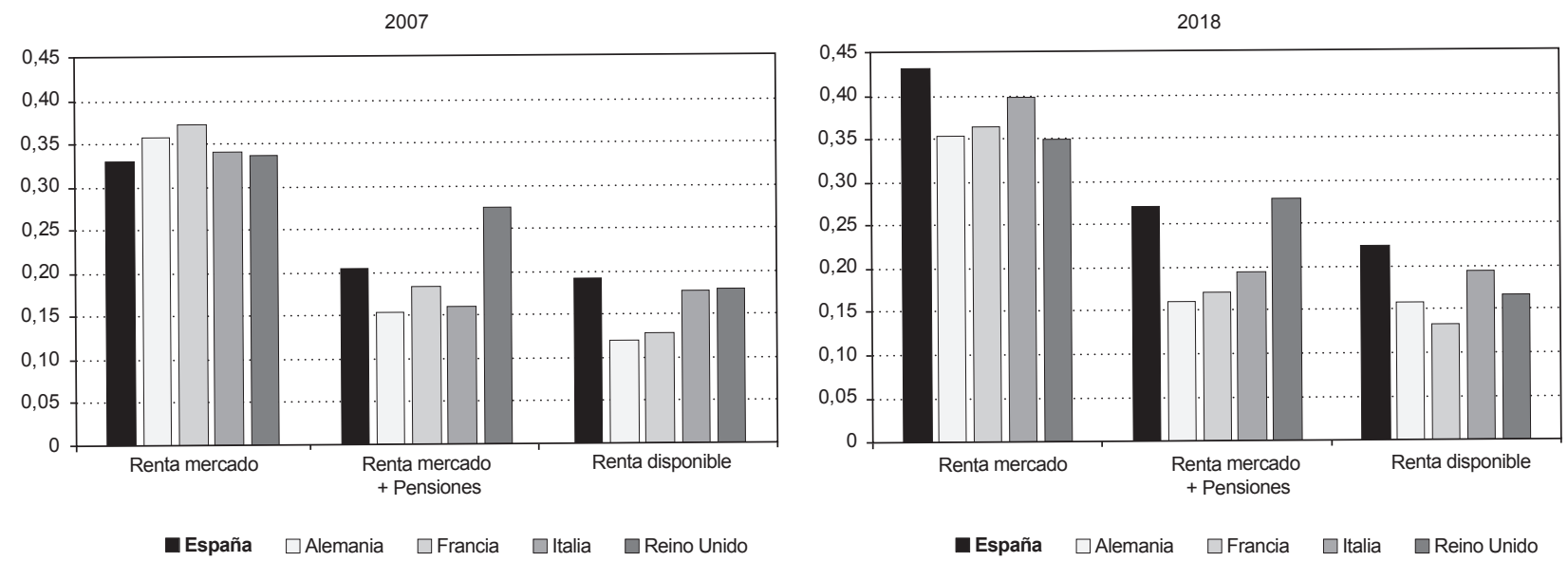

NOTA: La renta de mercado incluye salarios, ingresos del trabajo por cuenta propia, rentas de capital y otros ingresos. La renta disponible incluye renta original y todas las prestaciones monetarias del sistema (contributivas y no contributivas) y deduce las cotizaciones sociales y los impuestos personales directos.

FUENTE: Euromod I 1.0+.

trabajo autónomo, rentas de capital y otros ingresos), incluso si incluimos las pensiones contributivas, lo que reducía la visibilidad de que nuestro sistema de prestaciones e impuestos tenía una menor capacidad redistributiva y que no conseguía reducir las desigualdades tanto como los de otros países (Cantó, 2013). En consecuencia, cuando llegó la recesión y crecieron tanto el desempleo como el subempleo, la creciente desigualdad de rentas de mercado dejó ver que nuestro estado del bienestar era débil y que sin reformas progresivas estábamos abocados a colocarnos a la cabeza de la desigualdad de renta disponible en el conjunto de los países de la UE.

Si nos centramos en la parte más baja de la distribución y analizamos los cambios en el riesgo de pobreza en estos cinco países antes y después del proceso recesivo, las conclusiones son muy similares (Gráfico 3). En España e Italia tenemos sistemas menos capaces de reducir el riesgo de pobreza y las prestaciones más efectivas para reducirlo son las pensiones contributivas, mientras que el resto de transferencias tienen un papel muy limitado. En Alemania y Francia, estados del bienestar también basados en cotizaciones, las pensiones contributivas son también muy eficaces en reducir la pobreza, pero en Francia otras prestaciones también contribuyen de forma importante a reducir este riesgo. En Reino Unido el riesgo de pobreza es mayor que en Francia y Alemania y no son las pensiones contributivas sino otras prestaciones, a menudo focalizadas en los más pobres, las más eficaces en reducirlo.

En los Gráficos 4 y 5 analizamos los efectos redistributivos globales de todos los sistemas y vemos que, en su conjunto, tanto el sistema francés como el alemán son los más efectivos para reducir la desigualdad de renta disponible de las familias. El alemán, además, lo hace de manera muy estable en toda la última década, consiguiendo siempre reducir el índice de Gini por encima del $45 \%$, mientras que el francés ha oscilado 


\section{GRÁFICO 4}

\section{EFECTO REDISTRIBUTIVO GLOBAL DEL SISTEMA DE PRESTACIONES E IMPUESTOS EN CINCO PAÍSES DE LA UNIÓN EUROPEA \\ (En \% de reducción del índice de Gini)}

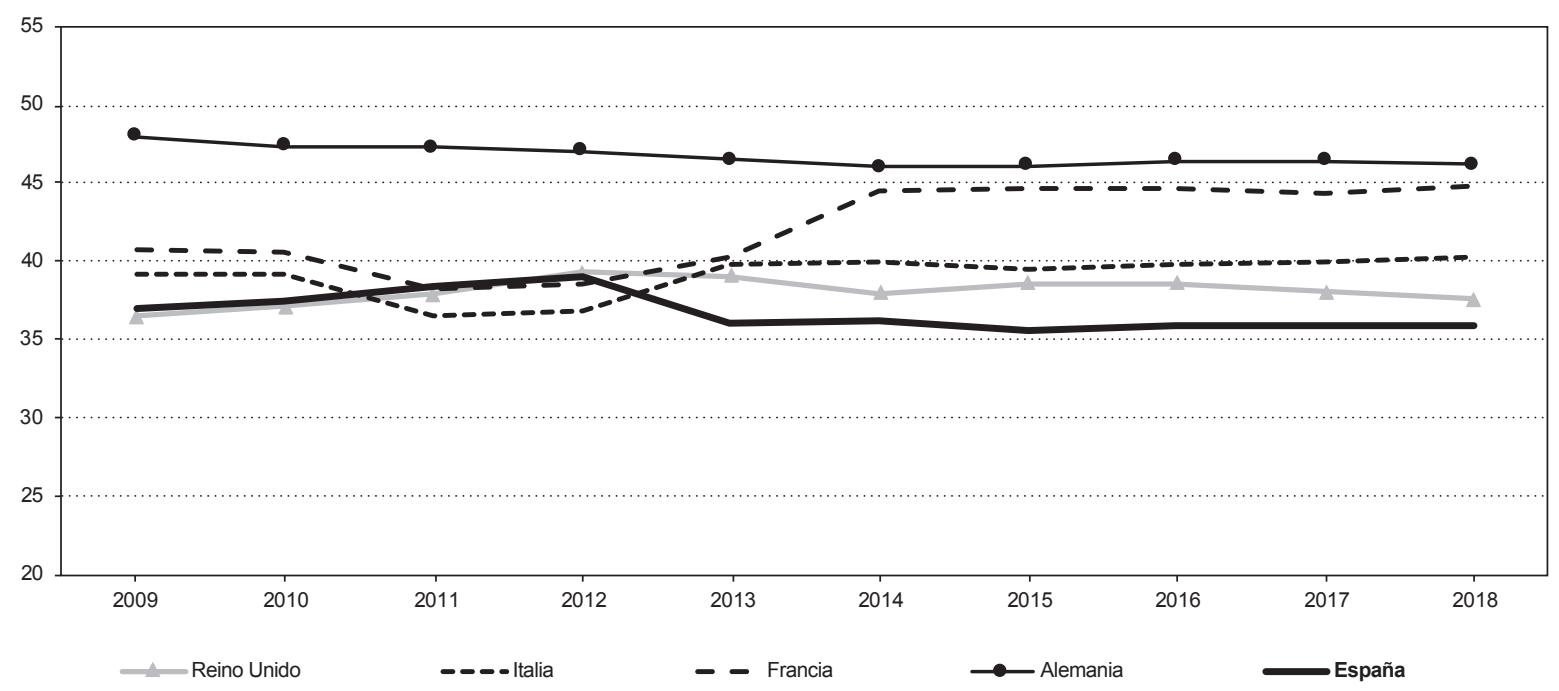

FUENTE: Euromod I 1.0+.

entre el 40 y el $45 \%$. El italiano y el británico son algo menos potentes y solo consiguen reducir la desigualdad medida con este índice en un $38 \%$. Finalmente, el sistema español es el más débil de todos y alcanza una capacidad redistributiva de alrededor de un $35 \%$, con una ligera mejora en 2012, uno de los peores momentos de la recesión.

Y, ¿a qué se debe que nuestro sistema no consiga reducir las desigualdades de la renta de mercado como lo hacen los sistemas de estos otros cuatro países? ¿a la falta de capacidad redistributiva de todas las prestaciones contributivas? ¿a la incapacidad de las no contributivas de proteger a los más vulnerables? ¿o al limitado papel de los impuestos directos? Las respuestas a estas preguntas son muy relevantes si pretendemos identificar hasta qué punto existe una brecha generacional en la capacidad de nuestro sistema para proteger a los hogares de distintas generaciones de la vulnerabilidad y la pobreza. Parece claro que un sistema basado en elementos contributivos y centrado en redistribuir en la vejez no puede ser muy efectivo cuando las generaciones más jóvenes se emancipan y cuando su historial de cotizaciones al sistema se ha debilitado tanto durante las últimas dos décadas, debido al aumento de la precariedad en sus condiciones laborales.

Como revela el Gráfico 5 nuestro sistema es menos redistributivo que otros, por la falta de capacidad redistributiva de otras prestaciones distintas de las pensiones. Si no las tenemos en cuenta, España e Italia son los dos países que tienen un sistema más débil para compensar las desigualdades de mercado. Es decir, en los hogares que no las reciben, la intervención del Estado reduce solo en un 20 \% las desigualdades primarias, lo que resulta ser la mitad que Reino Unido y un cuarto menos que Francia o Alemania. Sí parece claro, sin embargo, que de 2011 a 2014, durante los peores años de la recesión, algunas prestaciones de nuestro sistema protegieron mejor a los más 


\section{GRÁFICO 5}

\section{EFECTO REDISTRIBUTIVO DEL SISTEMA SIN LAS PENSIONES CONTRIBUTIVAS EN CINCO PAÍSES DE LA UNIÓN EUROPEA \\ (En \% de reducción del índice de Gini)}

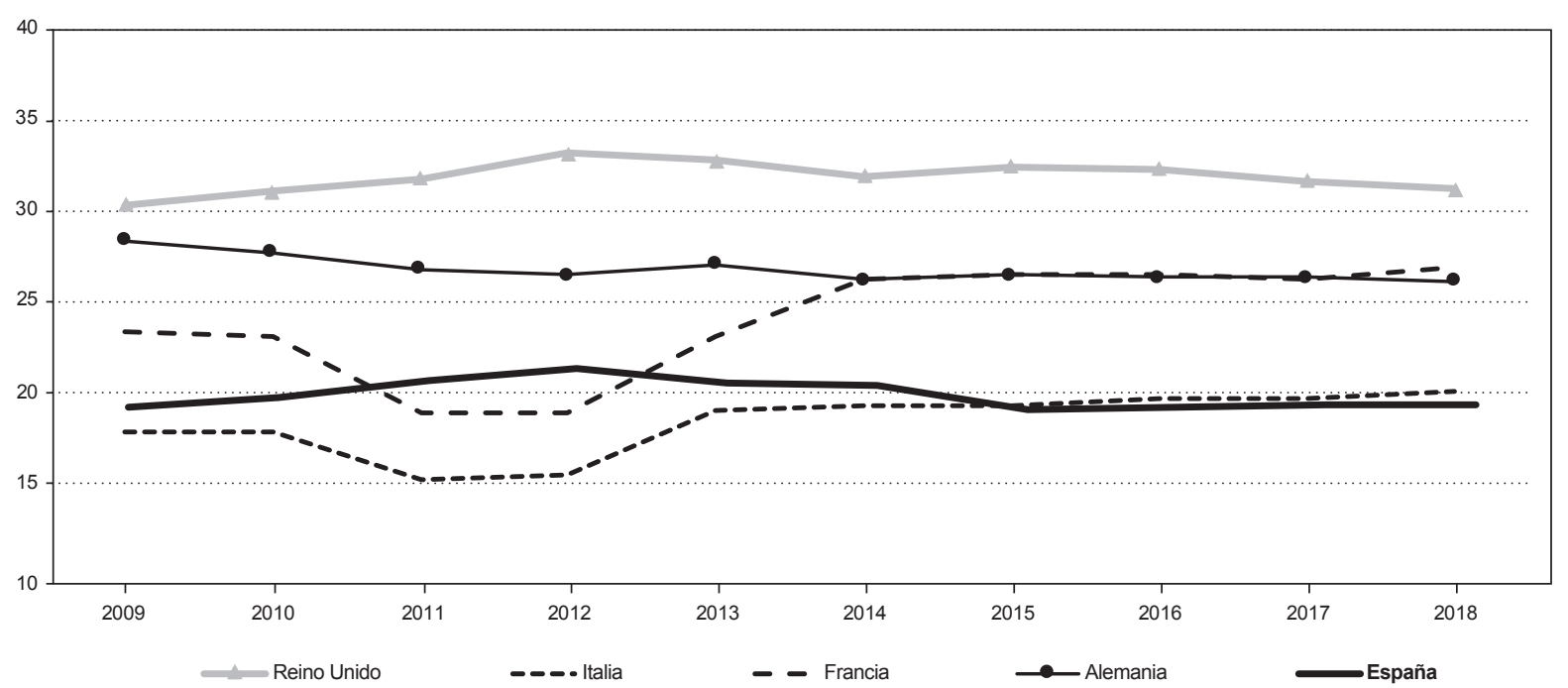

FUENTE: Euromod I 1.0+.

vulnerables que las de otros países, lo que hace ver que el sistema español tiene algunos elementos útiles para combatir la caída de rentas en los ciclos bajos, siempre que sean relativamente cortos.

Para comprender mejor qué herramientas resultan ser más determinantes para conseguir mayores cotas de redistribución de la renta disponible, individualizamos nuestra medida del efecto redistributivo por tipos de políticas (Gráfico 6). Los resultados indican que la cesta de políticas que permite reducir la desigualdad es variada, incluso entre los sistemas que clasificaríamos como inspirados en un sistema de seguro. Los más redistributivos son el francés y el alemán por su capacidad de comprimir ingresos tanto a través del impuesto personal sobre la renta, que reduce hasta un $50 \%$ más las desigualdades que en España, como a partir de sus pensiones contributivas y otras prestaciones. El sistema británico, algo más débil, consigue comprimir la renta disponible de su población fundamentalmente a través de prestaciones, algunas de ellas universales y muchas focalizadas en los más vulnerables, a la vez que, comparativamente, utiliza menos el impuesto sobre la renta personal como elemento redistribuidor, y reduce el índice de Gini en una cifra similar a la del impuesto español (diez puntos porcentuales).

Como apuntan Ayala et al. (2016), uno de los factores clave para la debilidad distributiva de las políticas de transferencias en España es el menor papel compensador de la última red de protección de los más pobres, las Rentas Mínimas de Inserción (RMI), que están fragmentadas por territorios y son extremadamente bajas en cuantía. Además, las prestaciones por hijo en España también son muy bajas y actualmente no suponen ni la cuarta parte de la prestación media por menor en los países de la Unión Europea (Ayala y Cantó, 2018; Cantó et al., 2014). Todo ello implica que poco más de la cuarta parte de la renta disponible de la familia española media sean prestaciones 


\section{GRÁFICO 6}

\section{EFECTO REDISTRIBUTIVO POR POLÍTICAS EN CINCO PAÍSES DE LA UNIÓN EUROPEA EN 2018}

(En \% de reducción del índice de Gini)

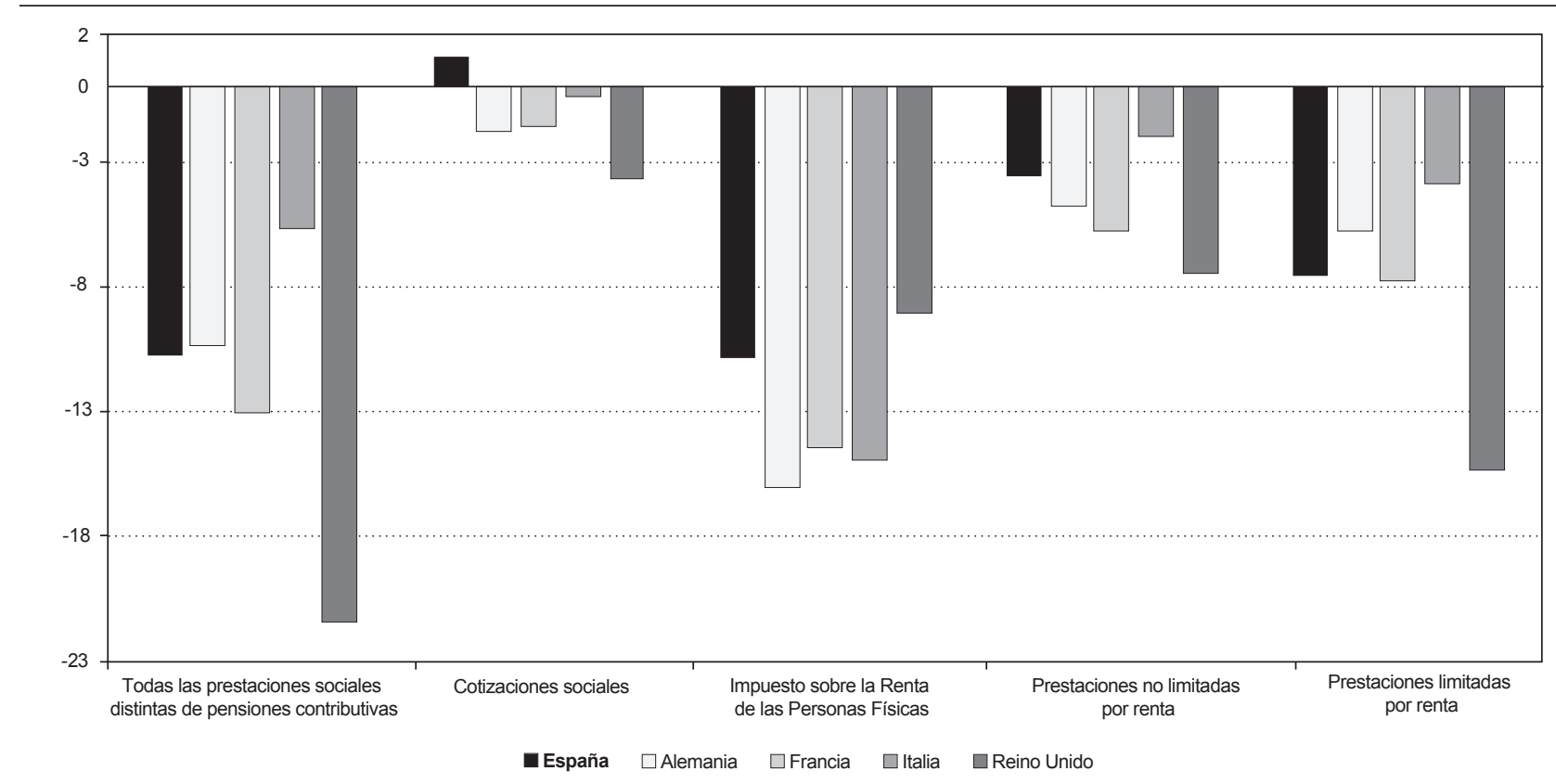

FUENTE: Euromod I 1.0+.

monetarias y cuatro quintas partes de estas sean pensiones contributivas. Adicionalmente, como subrayan Cantó (2013) y Avram et al. (2014), aunque la progresividad del impuesto sobre la renta español sea similar a la de otros países europeos, los tipos medios efectivos son los más bajos de los países de la eurozona, lo que lastra su capacidad redistributiva.

En resumen, en todos los sistemas basados en cotizaciones las pensiones contributivas son el elemento clave para la redistribución y reducen más de 20 puntos porcentuales el índice de Gini. En todo caso, hay también diferencias importantes entre los distintos modelos bismarckianos, ya que el impuesto personal sobre la renta en Francia y Alemania redistribuye bastante más que el español y las prestaciones universales (sin límite de renta) tienen también un papel mucho más importante que en nuestro país.

\section{El papel redistributivo de las políticas públicas ¿Hay una brecha generacional?}

Uno de los aspectos más preocupantes del fuerte incremento de las desigualdades en la mayoría de los países desarrollados en la última década es que, en muchos de ellos, se está abriendo una importante brecha generacional en los niveles de pobreza y vulnerabilidad económica (CJE, 2018). Como sería de esperar, esta brecha tiene consecuencias sobre las decisiones de emancipación de los jóvenes, condicionando tanto el establecimiento de nuevos hogares como su fecundidad y, aunque quizá sea menos evidente, también incide sobre las tasas de abandono escolar y sobre las posibilidades de búsqueda de empleo (lacovou y Aassve, 2007; Aassve et al., 2013). 


\section{GRÁFICO 7}

\section{EVOLUCIÓN DE LAS TASAS DE RIESGO DE POBREZA POR GRUPOS DE EDAD EN CINCO PAÍSES EUROPEOS}

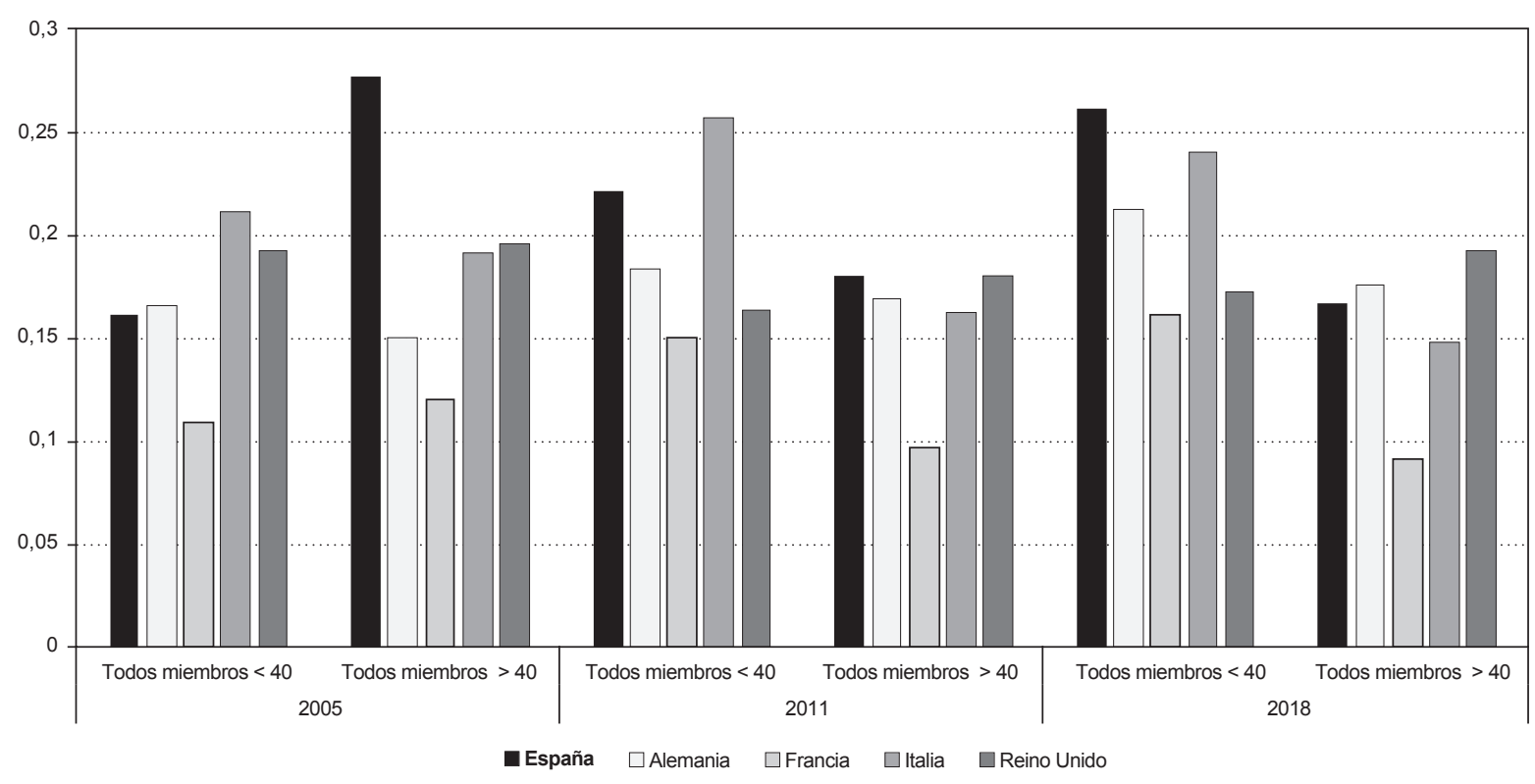

FUENTE: Euromod I 1.0+.

Con datos anteriores a la recesión, Ayllón (2015) concluye que muchos jóvenes escandinavos pasan un período corto de tiempo en la pobreza, mientras que en los países del sur de Europa, como España e Italia, la pobreza afecta a una menor proporción de jóvenes porque estos permanecen hasta edades avanzadas en el hogar parental, sin emanciparse. Lo más preocupante es que los episodios de pobreza de los jóvenes en el sur de Europa son mucho más duraderos que en otros países. Los jóvenes de países de Centroeuropa, como Alemania o Francia, y los anglosajones se colocarían en una posición intermedia entre lo que sucede en los países escandinavos y en los mediterráneos. Esta autora también concluye que la transición al empleo a principios de siglo estaba íntimamente relacionada con la emancipación en muchos países de la Unión Europea, y de hecho así era tanto en España e Italia como en Francia y Alemania, mientras que en Reino Unido y en Escandinavia esta relación era algo más débil.

En el Gráfico 7 podemos apreciar que las personas que vivían en hogares jóvenes en nuestro país en 2005 tenían un riesgo de pobreza más de diez puntos porcentuales inferior al de los que vivían en hogares en los que todos los miembros superaban los 40 años. Desde la llegada de la recesión en 2008 esto cambió y se ha ido abriendo progresivamente una brecha generacional en el riesgo de pobreza, que ha seguido creciendo hasta hoy. La brecha entre la tasa de riesgo de pobreza de los que viven en hogares en los que todos los miembros son menores de 40 años y los que viven en hogares en los que todos los miembros son mayores de esa edad es creciente en todos los países, excepto en Reino Unido. El país en el que esta diferencia 
es mayor es España, incluso superando a Italia. Una de cada cuatro personas que vive en hogares jóvenes en nuestro país es pobre, mientras que eso solo le sucede a una de cada seis de las que viven en hogares en los que todos tienen más de 40 años.

El profundo cambio en las diferencias de riesgo de pobreza por edades de la última década está ligado a tres factores principales: a que los trabajadores jóvenes ya sufrían tradicionalmente tasas de desempleo mucho más altas que los mayores, a que sus salarios mensuales (por salario/hora o por horas de trabajo) ahora son también mucho más bajos que los de los mayores $\mathrm{y}$, finalmente, a que el sistema de prestaciones e impuestos tiene menos capacidad para redistribuir los ingresos de las cohortes más jóvenes.

A partir de los datos de la Encuesta de Población Activa (INE, 2019), comprobamos que las tasas de desempleo de los más jóvenes han sido superiores a las de los mayores desde hace más de dos décadas. Actualmente la tasa media de desempleo de los menores de 40 años es un $54 \%$ más alta que la de los mayores de esa edad, con una tendencia nuevamente creciente desde principios de 2018, lo que indica que este es uno de los factores que contribuye y contribuirá a que los hogares formados solo por jóvenes tengan un riesgo de pobreza significativamente mayor que el resto. Según los datos de la Encuesta de Estructura Salarial (INE, 2019), la brecha salarial entre jóvenes y mayores ha aumentado significativamente en la última década. La diferencia salarial mensual media entre los ocupados menores de 40 años y los de 45 a 49 años se situaba antes de la recesión entre un 7 y un $45 \%$, dependiendo de la edad, en contra de los más jóvenes. Esta brecha se ha ido ampliando paulatinamente y en 2016 está entre un 11 y un $55 \%$. En la línea de lo que concluyen Ayala et al. (2019), lo más plausible es que estas diferencias salariales estén ligadas a la mayor incidencia de subempleo por falta de horas de trabajo entre los jóvenes, es decir, a que no consiguen trabajar el número de horas que desean, más que a un aumento en la diferencia en los salarios reales por hora entre distintas cohortes.
Profundicemos ahora en el tercer factor de riesgo: la capacidad protectora del sistema de prestaciones e impuestos para las diferentes cohortes de edad. En el Gráfico 8 presentamos resultados del porcentaje de reducción de la desigualdad de ingresos, medida con el índice de Gini antes y después de impuestos y prestaciones (incluidas las pensiones contributivas) en los cinco países considerados. Como se aprecia claramente, todos los sistemas redistribuyen algo más las rentas de las cohortes mayores que las de las más jóvenes, pero hay diferencias importantes entre países. En España e Italia las diferencias entre una y otra cohorte de edad son mayores: estos sistemas reducen la desigualdad en casi un $60 \%$ a las personas que viven en hogares en los que todos sus miembros son mayores de 40 años, mientras que lo hacen solamente en un 15 o un $17 \%$ en los hogares solo con jóvenes, y un $25 \%$ en los hogares con jóvenes y mayores (familias con hijos, en su mayoría).

Las diferencias entre cohortes son algo menores en Francia y Alemania y mucho menores en Reino Unido, donde consiguen reducir la desigualdad medida con el índice de Gini de forma bastante equitativa entre generaciones: un $43 \%$ entre los mayores y un $37 \%$ entre los jóvenes. Estos resultados están íntimamente relacionados con el papel que juegan las pensiones contributivas. Estas pensiones, que tienen como principal objetivo redistribuir rentas a lo largo del ciclo vital, están diseñadas de tal manera que también comprimen las rentas de una parte muy importante de la población y son actualmente el instrumento más potente para reducir la desigualdad en todos los países analizados, aunque lo hacen en mucha menor medida en el sistema más asistencialista representado por Reino Unido que en el resto.

Las otras políticas de transferencias tienen efectos redistributivos distintos según los países (Gráficos 9 y 10). La menor capacidad de redistribuir las rentas de los más jóvenes en países como España e Italia se explica porque la capacidad redistributiva de otras transferencias distintas de las pensiones contributivas es pequeña (entre todas ellas no superan 


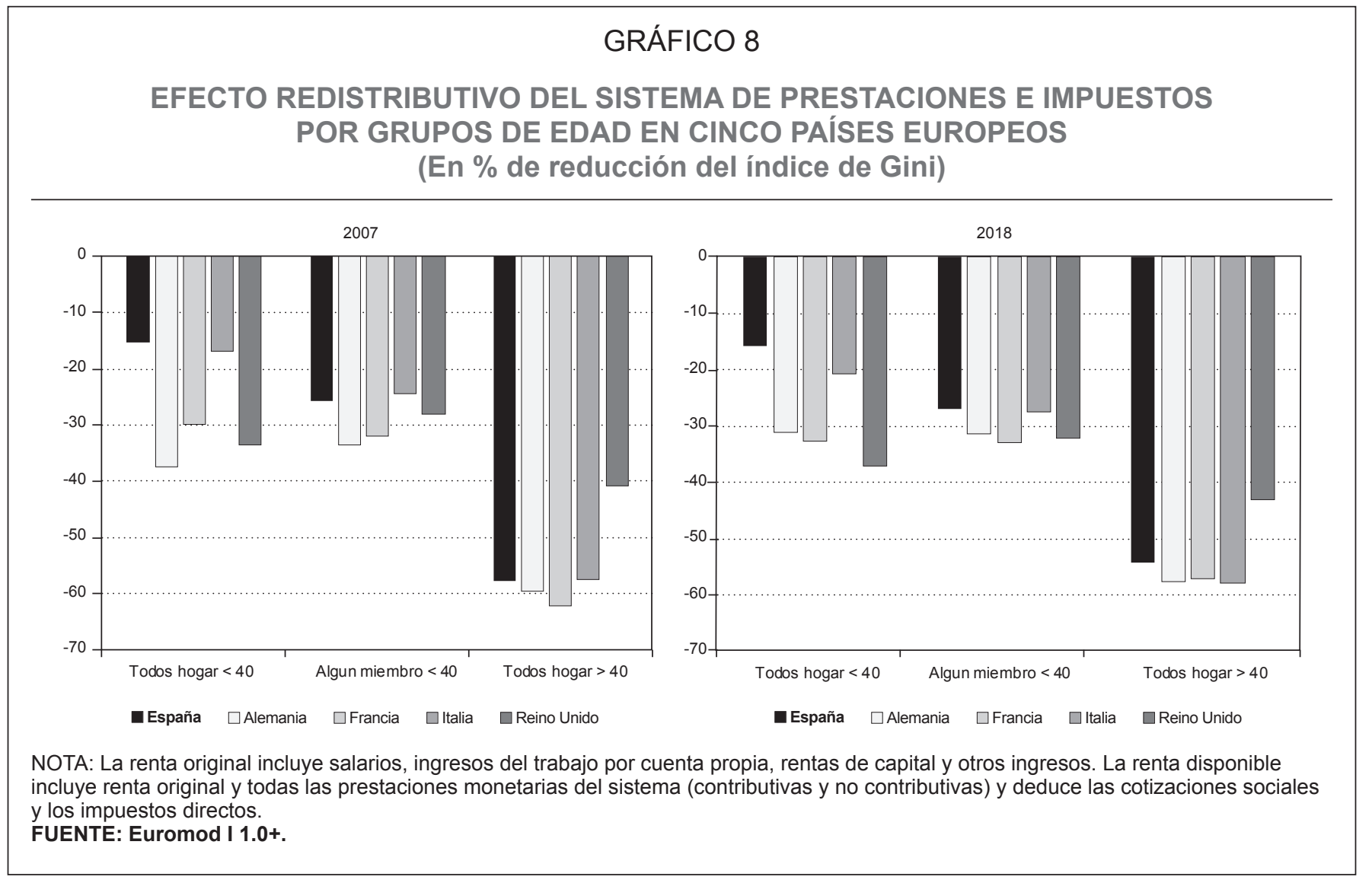

los 12 puntos porcentuales de reducción del índice de Gini), mientras que en Francia, Alemania y especialmente en Reino Unido son mucho más potentes, sobre todo para reducir la desigualdad y la pobreza entre los jóvenes. En el caso español, estas transferencias son más efectivas para reducir la desigualdad de aquellos que viven en hogares donde todos los miembros superan los 40 años (12 puntos porcentuales) y no tanto para aquellos que viven en hogares en los que todos son menores de esa edad (8 puntos porcentuales), lo que da una idea de la falta de cobertura de necesidades de las generaciones más jóvenes. En todos los demás países, excepto Italia, estas transferencias reducen más la desigualdad de la cohorte más joven que la de los mayores: 16 puntos porcentuales versus 7, 21 versus 6, o 29 versus 17 en Alemania, Francia y Reino Unido respectivamente.
Si diferenciamos entre prestaciones focalizadas (con límite de renta) y las que no lo son (sin límite de renta o universales), comprobamos que en nuestro país las primeras son mucho más efectivas que las segundas para reducir el riesgo de pobreza de los más jóvenes (Gráfico 10). Las primeras incluyen las rentas mínimas de las comunidades autónomas, el Programa de Activación para el Empleo (PAE) y las prestaciones por hijo a cargo, además de las pensiones no contributivas, mientras que las segundas están más ligadas a las cotizaciones en el mercado de trabajo e incluyen sobre todo prestaciones por desempleo contributivas. Lo contrario sucede entre los mayores: las prestaciones sin límite que dependen de cotizaciones previas y que tienen en cuenta la situación familiar son las que más ayudan a reducir su riesgo de pobreza. 


\section{GRÁFICO 9}

\section{EFECTO REDISTRIBUTIVO POR POLÍTICAS (EXCEPTO PENSIONES CONTRIBUTIVAS) Y GENERACIONES EN CINCO PAÍSES EUROPEOS, 2018}

(En \% de reducción índice de Gini)
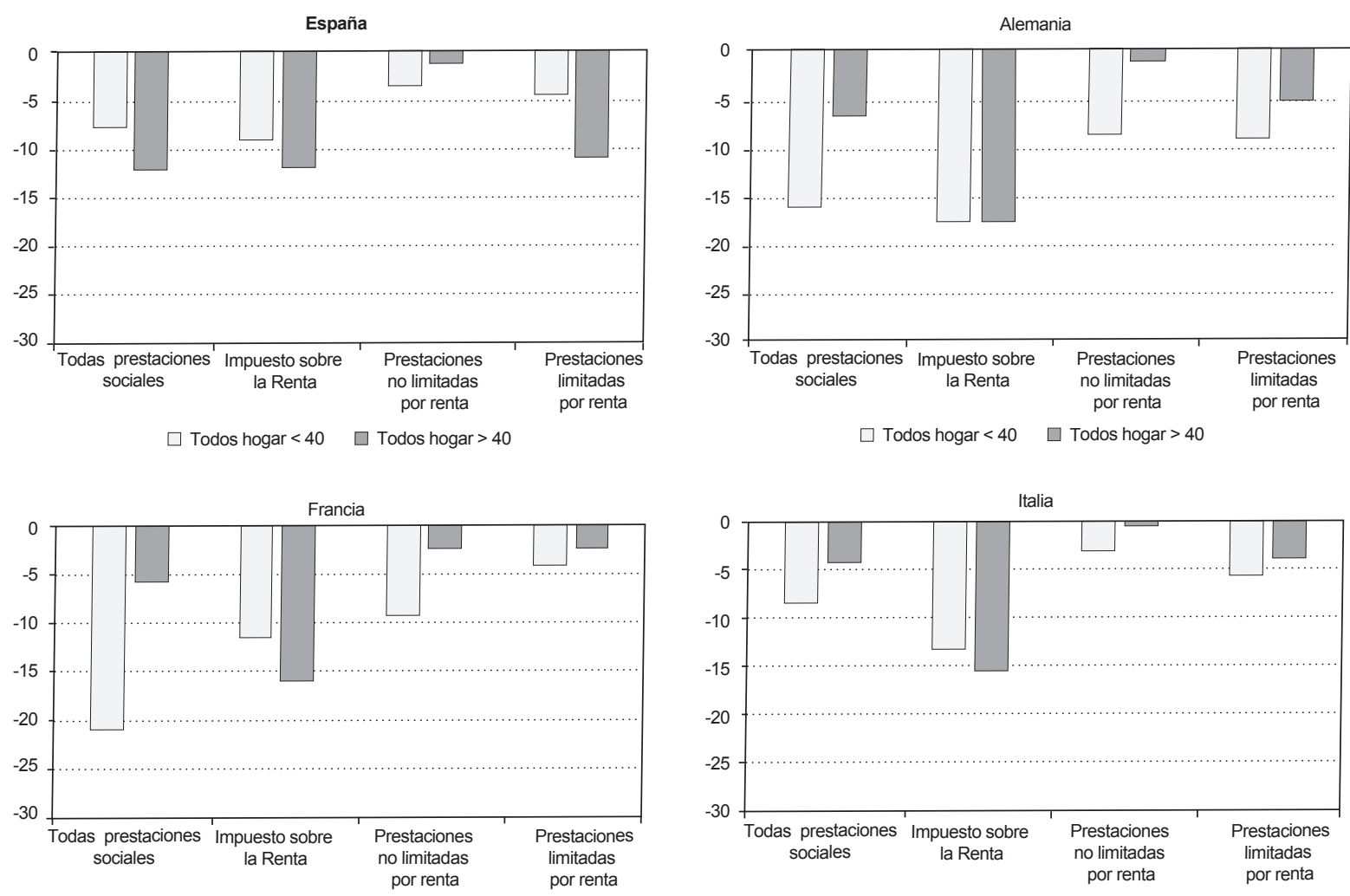

$\square$ Todos hogar $<40 \quad \square$ Todos hogar $>40$

$\square$ Todos hogar $<40 \quad \square$ Todos hogar $>40$

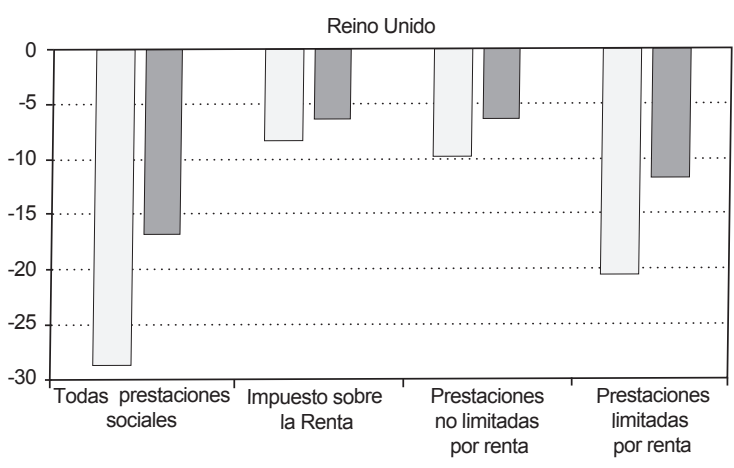

$\square$ Todos hogar $<40 \quad \square$ Todos hogar $>40$

NOTA: Las prestaciones sociales no incluyen las pensiones contributivas. Las prestaciones no limitadas por renta incluyen todas las prestaciones contributivas y universales, y las limitadas por renta incluyen todas las prestaciones focalizadas sobre los más pobres. Hay que tener en cuenta que estos cálculos no incluyen los efectos redistributivos cruzados entre políticas que suponen menos de un 5 \% de efecto redistributivo total en todos los países.

FUENTE: Euromod I 1.0+. 


\section{GRÁFICO 10}

CAPACIDAD DE REDUCIR LA POBREZA POR POLÍTICAS ENTRE JÓVENES Y MAYORES, 2018 (En \% de reducción de la tasa de riesgo de pobreza)

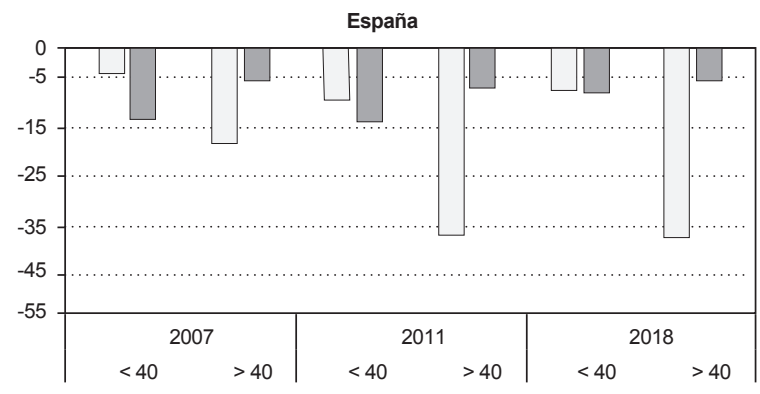

$\square$ Prestaciones sin límite de renta $\quad \square$ Prestaciones con límite de renta

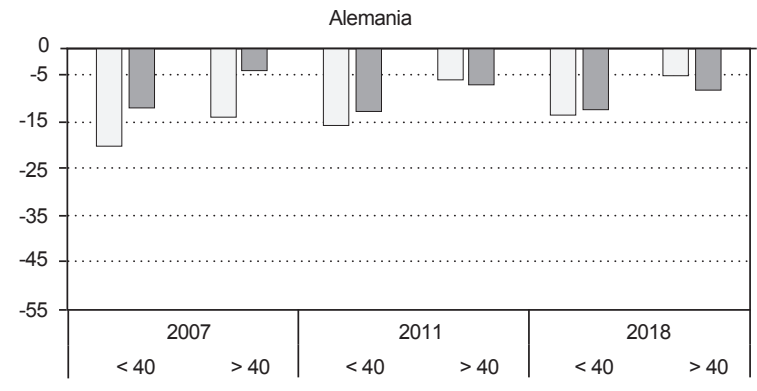

$\square$ Prestaciones sin límite de renta $\quad \square$ Prestaciones con límite de renta

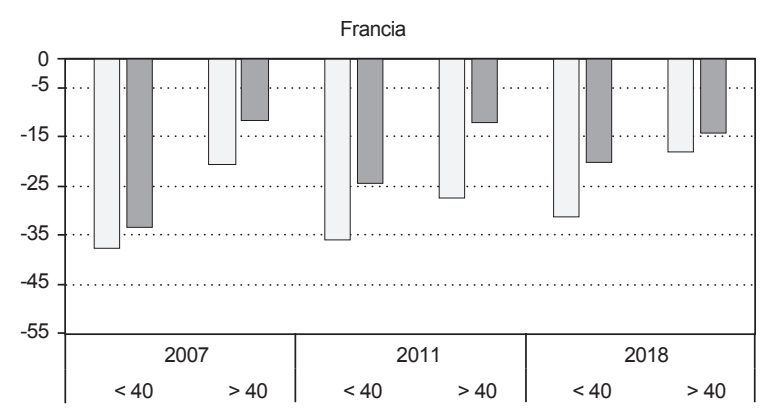

$\square$ Prestaciones sin límite de renta $\quad \square$ Prestaciones con límite de renta

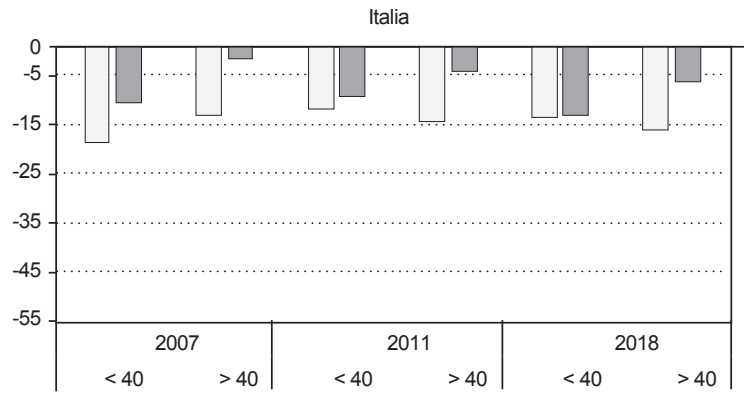

$\square$ Prestaciones sin límite de renta $\quad \square$ Prestaciones con límite de renta

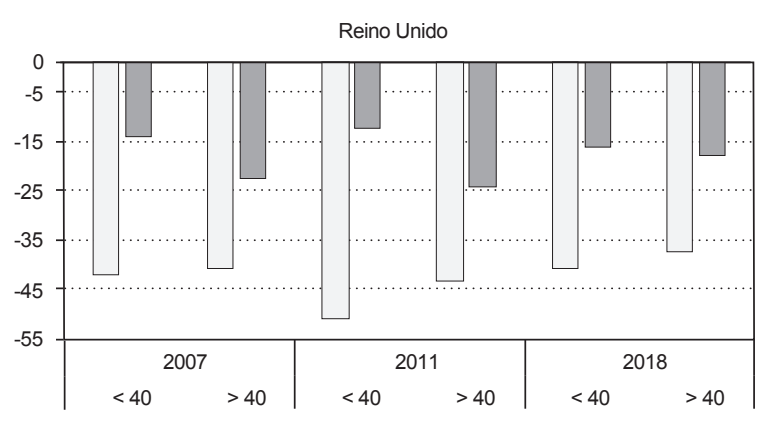

$\square$ Prestaciones sin límite de renta $\quad \square$ Prestaciones con límite de renta

NOTA: Las prestaciones sociales no incluyen las pensiones contributivas. Las prestaciones no limitadas por renta incluyen todas las prestaciones contributivas y universales y las limitadas por renta incluyen todas las prestaciones focalizadas sobre los más pobres. Hay que tener en cuenta que estos cálculos no incluyen los efectos redistributivos cruzados entre políticas que suponen menos de un 5 \% de efecto redistributivo total en todos los países.

FUENTE: Euromod I 1.0+. 
Francia y Reino Unido destacan por ser los dos países en los que las prestaciones distintas de las pensiones contributivas ayudan más a reducir la pobreza juvenil; en más de un $50 \%$ si consideramos todo tipo de prestaciones. A ello contribuyen especialmente las transferencias universales, es decir, sin límite de renta, que en el caso de Reino Unido reducen el riesgo de pobreza más del doble que las transferencias focalizadas entre los más jóvenes.

En resumen, las altas tasas de desempleo entre los jóvenes y el mayor deterioro de sus condiciones laborales son el primer elemento que contribuye al aumento de la brecha generacional de bienestar económico en muchos países europeos, lo que se traduce en altas tasas de riesgo de pobreza para los menores de 40 años. El segundo es la falta de políticas sociales correctoras, porque las reformas llevadas a cabo en los estados del bienestar bismarckianos, con un gran peso relativo de las prestaciones contributivas, aún no han conseguido equilibrar la balanza y están en un proceso de larga transición, desde el antiguo modelo de mediados del siglo XX, ligado al empleo, a un modelo más centrado en las necesidades de ingresos de las personas.

Los sistemas "à la Beveridge» como el de Reino Unido son los más equilibrados en términos generacionales, pero tienden a ser más débiles de forma global y, en conjunto, reducen menos la desigualdad que algunos de los bismarckianos más potentes, como el alemán o el francés que basan más su capacidad redistributiva en el impuesto directo sobre la renta y en el sistema de pensiones. En general, de estos dos, el sistema francés destaca por ser el que tiene prestaciones más eficaces para reducir la pobreza juvenil.

Dentro del grupo de los sistemas bismarckianos, los estados del bienestar mediterráneos, el español y el italiano, son los más débiles en cuanto a capacidad redistributiva se refiere, y son los que menos han conseguido avanzar en las últimas décadas en la transición hacia un modelo más equilibrado entre generaciones.

\section{Conclusiones}

En este artículo hemos comprobado que existe una brecha generacional de distinta dimensión en el papel que desempeñan las políticas públicas en la lucha contra la vulnerabilidad y la pobreza en cinco países europeos.

Nuestros resultados indican que los cuatro sistemas caracterizados por un estado del bienestar de corte más bismarckiano (el francés, el alemán, el italiano y el español) consiguen reducir algo menos las desigualdades y el riesgo de pobreza de las generaciones más jóvenes de lo que lo hace un sistema como el británico, de corte más asistencialista. Parecería entonces que los primeros, al haber asignado un mayor peso redistributivo a las pensiones contributivas, no han desarrollado suficientemente otras políticas de transferencias dirigidas a cohortes más jóvenes. El sistema británico es el más equilibrado en términos generacionales pero, en conjunto, reduce menos la desigualdad y la pobreza que algunos de los bismarckianos más potentes, como el alemán o el francés. Estos dos últimos utilizan más la capacidad redistributiva del impuesto directo sobre la renta a la vez que, como el británico, tienen prestaciones universales y focalizadas bastante efectivas. En general, de estos, es el francés el que destaca por tener unas prestaciones, tanto universales como focalizadas, eficaces en reducir el riesgo de pobreza juvenil.

Entre los cuatro países con sistemas más bismarckianos, se observan también algunas diferencias importantes: España e Italia tienen mayor brecha por cohortes de edad que Francia o Alemania y, además, su capacidad para compensar las desigualdades de mercado es mucho más limitada. En el caso español, el menor papel compensador de la última red de ingresos garantizados, las RMI y la baja cuantía de las prestaciones por hijo a cargo limitan la capacidad redistributiva del sistema para las cohortes más jóvenes. En todo caso, y a pesar de su limitada capacidad, estas políticas focalizadas son actualmente las 
más efectivas para reducir el riesgo de pobreza de los jóvenes, por las dificultades que tienen para acceder a otras transferencias sin límite de renta (o universales) que están ligadas a cotizaciones previas como las prestaciones por desempleo contributivas.

En definitiva, las crecientes dificultades de los jóvenes para acceder a un empleo de calidad que les permita tener historiales de cotización estables y el constante deterioro de sus condiciones laborales están aumentando notablemente su riesgo de vulnerabilidad y de pobreza. En países como España es indudable que se deben introducir reformas en el sistema de prestaciones e impuestos, que permitan empezar a transitar hacia un modelo de estado de bienestar más centrado en las necesidades de ingresos de las personas y sus familias que en el historial laboral individual. Esto se debe abordar con sensatez y sin fracturar otros elementos clave del sistema como las pensiones y las prestaciones por desempleo contributivas que, a día de hoy, siguen siendo las transferencias más importantes para reducir la desigualdad de rentas y el riesgo de pobreza.

\section{Referencias bibliográficas}

Aassve, A., Cottini, E. and Vitali, A. (2013). Youth prospects in a time of economic recession. Demographic Research, 29(36).

Avram, S., Figari, F., Leventi, C., Levy, H., Navicke, J., Matsaganis, M., Militaru, E., Paulus, P., Rastrigina, O. and Sutherland, H. (2013). The distributional effects of fiscal consolidation in nine EU countries. Euromod Working Paper Series (EM2/13). Colchester: University of Essex.

Avram, S., Levy, H. and Sutherland, H. (2014). Income redistribution in the European Union. IZA Journal of European Labor Studies, 3-22. June.

Ayala, L., Arranz, J. M., García-Serrano, C. y Martínez-Virto, I. (2016). El sistema de garantía de ingresos en España: tendencias, resultados y necesidades de reforma. Ministerio de Sanidad, Servicios Sociales e Igualdad. Gobierno de España.

Ayala, L. and Cantó, O. (2018). The driving forces of rising inequality in Spain: is there more to it than a deep worsening of low-income households' living standards? En B. Nolan
(Ed.), Inequality and inclusive growth in rich countries: shared challenges and contrasting fortunes, capítulo 10. Oxford: Oxford University Press.

Ayala, L., Cantó, O., Navarro, C., Martínez, R. y Romaguera, M. (2019). Mercado de trabajo. Informe Observatorio Social La Caixa sobre Necesidades Sociales. Barcelona: en prensa.

Ayala, L., Martínez, R. y Sastre, M. (2006). Familia. Infancia y privación social. Madrid: Fundación FOESSA.

Ayllón, S. (2015). Youth poverty, employment and leaving the parental home in Europe. Review of Income and Wealth, 61(4), diciembre, 651-676.

Bargain, O., Callan, T., Doorley, K. and Keane, C. (2017). Changes in income distributions and the role of tax-benefit policy during the great recession: an international perspective. Fiscal Studies, 38 (4), 559-585.

Cantó, O. (2013). El efecto redistributivo del impuesto sobre la renta y las prestaciones monetarias ante el incremento de la desigualdad de rentas. Presupuesto y Gasto Público, (71), 153-170, ISSN: 0210-5977.

Cantó, O., Ayala, L., Adiego, M., Levy, H. and Paniagua, M. M. (2014). Going regional: the effectiveness of different tax-benefit policies in combating child poverty in Spain. En G. Dekkers, M. Keegan y C. O'Donoghue (Eds.), New Pathways in Microsimulation, capítulo 12, 183-202. Ashgate.

CJE. (2018). Estudio sobre pobreza juvenil, mayo. Consejo de la Juventud de España.

Corak, M., Curtis, L. J. and Phipps, S. (2011). Economic Mobility, Family Background, and the Well-being of Children in the US and Canada, capítulo 3. En T. Smeeding, R. Erikson, and M. Jantti, (Eds.), Persistence, Privilege and Parenting: the Comparative Study of Intergenerational Mobility. The Russell Sage Foundation.

De Agostini, P., Paulus, A. and Tasseva, I. (2016). The effect of tax-benefit changes on the income distribution in 20082015. Euromod Working Paper 6/16. Colchester: University of Essex.

Ermisch, J., Francesconi, M. and Pevalin D. J. (2001). Outcomes for children of poverty. Research Report (158). London: Department for Work and Pensions.

lacovou, M. and Aassve, A. (2007). Youth poverty in Europe. Joseph Rowntree Foundation.

INE (2019). Encuesta de Población Activa y Encuesta de Estructura Salarial. Madrid.

OECD (2015). In it together: why less inequality benefits all. París: OECD Publishing.

Paulus, A. and Tasseva, I. (2018). Europe through the crisis: discretionary policy changes and automatic stabilisers. Euromod WP, (16/18). 
En el próximo número de

Información Comercial Española. Revista de Economia

\section{Multinacionales \\ en un cambiante contexto internacional}

Expansión internacional de las empresas multinacionales. Estructura y naturaleza institucional

La decisión de inversión directa en el exterior: naturaleza, factores y condicionantes

Digitalización y multinacionales

Governance Structure and location in the information and digital age

El crecimiento de las plataformas digitales en el escenario internacional

Global Value Chains and Local Systems: a tale of two sides of the coin

Multinacionales emergentes

La expansión interregional en Iberoamérica: la creación de valor a través de filiales trampolín

Conocimiento para la internacionalización e inversión exterior de la pyme: un modelo descriptivo de la naturaleza y alcance de la relación

Recent policy developments regarding foreign investment
Últimos números publicados:

La crisis financiera.

Diez años después

La Mejora de la

Regulación

Números en preparación:

Economía de

la educación y política educativa

Startups, nuevo foco de las políticas públicas de atracción de talento, know how y transferencia de conocimiento. El caso de España e ICEX - Invest in Spain 